\title{
Aplicabilidade do teste da habilidade de atenção auditiva sustentada - THAAS em campo livre
}

\author{
Applicability of the free field Sustained Auditory Attention Ability Test (SAAAT)
}

\author{
Mariza Ribeiro Feniman', Ariane Cristina Sampaio Rissatto², José Roberto Pereira Lauris³, Maria Fernanda Capoani Garcia Mondelli. \\ 1) Pós-Doutorado em Audiology - Univeristy of Cincinnati-Ohio-USA. Chefe de Departamento de Fonoaudiologia-FOB-USP. Professora Titular do Departamento de \\ Fonoaudiologia da FOB/USP. \\ 2) Mestre em Fonoaudiologia pela FOB/USP. Fonoaudióloga. \\ 3) Liv re docência. Professor Associado do Departamento de Odontopediatria, Ortodontia e Saúde Coletiva da Faculdade de Odontologia de Bauru da Universidade de \\ São Paulo, FOB/USP. \\ 4) Doutora em Distúrbios da Comunicação pelo HRAC/USP. Professora Doutora do Departamento de Fonoaudiologia da FOB/USP. \\ Instituição: Faculdade de Odontologia de Bauru - Universidade de São Paulo. \\ Bauru / SP - Brasil. \\ Endereço para correspondência: Mariza Ribeiro Feniman - Alameda Octávio Pinheiro Brizola 9-75 - Vila Universitária - Bauru / SP - Brasil - CEP: $17012-901$ - E-mail: \\ mfernandamondelli@hotmail.com \\ Artigo recebido em 28 de abril de 2011. Artigo aprovado em 25 de junho de 2011.
}

\section{RESUMO}

Introdução: A atenção é uma função neuropsicológica subjacente a todos os processos cognitivos. A deficiência auditiva compromete o desenvolvimento normal da criança, alterando diversas habilidades auditivas, incluindo a atenção. Objetivo: comparar o desempenho de crianças no Teste da Habilidade de Atenção Auditiva Sustentada-THAAS, no que se refere às diferentes formas de aplicação (fones auriculares e campo livre), gênero e, ordem de aplicação.

Método: participaram 40 crianças ( 7 anos) voluntárias com desenvolvimento típico, divididas em dois grupos: G1 e G2, compostos de 20 crianças cada. A aplicação do THAAS no G1 se deu primeiramente com fones auriculares e em seguida em campo livre e no G2 o processo foi inverso. A avaliação constituiu-se em: questionário específico, testes auditivos e aplicação do THAAS.

Resultados: Não houve diferença significante quanto ao gênero. Para o THAAS com fones, o G1 apresentou maior quantidade de erros de desatenção e pontuação total. Para o THAAS em campo houve uma diferença significante do G2 para o decréscimo de vigilância. Quanto à forma de aplicação, o G1 demonstrou um número maior de erros quando foi utilizado fones. O G2 não demonstrou diferença.

Conclusão: Houve viabilidade na aplicação do THAAS em Campo Livre, podendo ser adotado os mesmos valores normativos usados para o modo convencional de avaliação. Palavras-chave: audição, atenção, criança.

\section{SUMMARY}

Introduction: The attention is an underlying neuropsychology function to all the cognitive processes. The auditory deficiency compromises the normal development of the child, modifying diverse auditory abilities, including the attention.

Objective: to compare the performance of children in the Test of the Ability of Auditory Attention Support, as for the different forms of application (auricular phones and free field), sort and, application order.

Method: 40 children ( 7 years old) voluntary with typical development had participated, divided in two groups: G1 and G2, composites of 20 children each. The application of the THAAS in the G1 if gave first with auricular phones and after that in free field and the G2 the process was inverse. The evaluation consisted in: specific questionnaire, auditory tests and application of the THAAS.

Results: It did not have significant difference how much to the sort. For the THAAS with phones, the G1 presented greater amount of errors of carelessness and total punctuation. For the THAAS in field it had a significant difference of the G2 for the monitoring decrease. How much to the application form, the G1 demonstrated a bigger number of errors when it was used phones. The G2 did not demonstrate difference.

Conclusion: It had viability in the application of the THAAS in Free Field, being able to be adopted the same used normative values for the conventional way of evaluation.

Keyword: hearing, attention, child. 


\section{INTRODUÇÃO}

A existência de uma deficiência auditiva por si só compromete o desenvolvimento normal de uma criança, uma vez que a privação sensorial pode gerar alterações em diversas habilidades auditivas, incluindo a atenção (1). Assim, ainda que crianças portadoras desse tipo de deficiência estejam devidamente inseridas num processo de reabilitação auditiva, seja fazendo uso de aparelho de amplificação sonora individual (AASI) ou do implante coclear (IC), além da fonoterapia, essas crianças podem estar sujeitas ainda a outros problemas, como aqueles relacionados à atenção auditiva.

Identificar e prestar atenção a aspectos acústicos e fonéticos dos padrões linguísticos é essencial para a aquisição e para o desenvolvimento da linguagem, tanto para crianças ouvintes normais quanto para as deficientes auditivas. Com a habilidade de atenção deficitária, estes aspectos podem se encontrar prejudicados, interferindo no desempenho escolar, cognitivo e social da criança (2).

A atenção é uma função neuropsicológica básica que está subjacente a todos os processos cognitivos. Pode ser definida como a capacidade do indivíduo selecionar e focalizar seus processos mentais em algum aspecto do ambiente interno ou externo, respondendo predominantemente aos estímulos que lhe são significativos e inibindo respostas aos demais estímulos $(3,4)$.

A literatura relata que a atenção sustentada e a vigilância são alguns dos processos que caracterizam a habilidade de atenção. A atenção não constitui um processoúnico.

A atenção sustentada é definida como a capacidade de manter o foco atencional em um determinado estímulo, por um período de tempo, para executar uma tarefa $(4,5)$. A vigilância é a habilidade para manter-se preparado para um sinal intermitente.

A utilização do Teste de Habilidade de Atenção Auditiva Sustentada - (THAAS) (6,7), realizada em seu modo convencional, com fones auriculares, tem se mostrado uma eficiente e promissora ferramenta para avaliar esta importante habilidade nas diferentes entidades clínicas, deficiência auditiva (8), fissura labiopalatina (9), síndrome velocardiofacial (10) e transtorno fonológico (11).

O THAAS é baseado no teste de desempenho contínuo, ACPT-Auditory Continuous Performance Test (12), que é empregado clinicamente para medir a atenção auditiva, assim, requer que o sujeito focalize e sustente a atenção durante toda a prova e responda para um estímulo alvo previamente especificado (13).

Com o intuito de avaliar esta importante habilidade auditiva em crianças que fazem uso de dispositivos eletrônicos, assim como àquelas que não são cooperadoras na utilização de fones auriculares, durante a realização de uma avaliação comportamental; ao longo de anos de vivência clínica com esta população, pensou-se em verificar a aplicabilidade do THAAS não apenas utilizando seu modo convencional.

O objetivo deste trabalho é comparar o desempenho de crianças no THAAS no que se refere às diferentes formas de apresentação, com fones auriculares (modo convencional) e em campo livre; ao gênero e, à ordem de aplicação, visando sua posterior aplicaçãoàquelas que não permitem a colocação de fones auriculares, que fazem uso de AASI ou de IC.

\section{MÉTODO}

Este trabalho foi desenvolvido na Clínica de Fonoaudiologia da Faculdade de Odontologia de Bauru da Universidade de São Paulo, tendo sido submetido à aprovação pelo Comitê de Ética em Pesquisa em Seres Humanos sob o parecer n 157/2007.

A fim de se selecionar a casuística para este estudo, ou seja, crianças de sete anos a sete anos e 11 meses, com ausência de queixa e/ou alteração auditiva, sem comprometimento intelectual, e sem histórico de desatenção, foi feito contato com pais de escolares, a fim de realizar convite a seus filhos, pertencentes a esta idade para participarem do estudo. Após o aceite as crianças foram alocadas ao acaso e pareadas o mais próximo possível quanto ao gênero.

A idade escolhida deve-se ao fato de ser uma idade de grande importância para o aprendizado, período de alfabetização, cuja habilidade de atenção faz-se essencial.

Após a leitura e a assinatura do Termo de Consentimento Livre e Esclarecido, os pais e/ou responsáveis foram submetidos ao questionário de atenção, visando obter informações quanto aos dados pessoais; quanto à presença de problemas neurológicos ou outras deficiências; quanto à saúde auditiva, aos aspectos comportamentais e acadêmicos relacionados à manifestação ou queixas de desatenção de sua criança.

Após, todas as crianças foram submetidas à inspeção visual do meato acústico, à imitanciometria, por meio da utilização do imitanciômetro GSITympstar, à Audiometria 
Tonal Liminar, utilizando o audiômetro Siemens SD50, com fones auriculares e em cabina acústica; ao Limiar de recepção de fala/SRT e à Audiometria em Campo Livre, realizada no audiômetro Clinical AudiometerAC40.

Considerou-se como critério de inclusão no presente estudo:

- Idade igual há sete anos a sete anos e 11 meses.

- Ausência de queixas auditivas ou afecção das vias aéreas superiores.

- Ausência de problemas neurológicos ou outras deficiências.

- Até sete questões assinaladas no questionário de atenção.

- Ausência de corpo estranho e/ou excesso de cerúmen na orelha externa.

- Curva Timpanométrica normal - Tipo A (14).

- Limiar audiométrico até 15 dBNA (15), com SRT compatível.

- Obtenção de um escore total de erros (pontuação total) igual ou inferior a 36 e decréscimo de vigilância menor que oito no THAAS (convencional).

Do total de 106 crianças atendidas, dados de 40 delas puderam ser computados neste trabalho, tendo em vista os critérios de inclusão estabelecidos. A participação dessas 40 crianças foi determinada como sendo um número amostral significativo para o desenvolvimento da pesquisa, sendo, portanto, este o número mínimo de crianças pré-determinado para participarem deste estudo.

Assim, foi realizado um estudo randomizado nas 40 crianças voluntárias, sendo 11 do gênero masculino e nove do feminino, tanto para o G1 como para o G2, aplicandose o THAAS em diferentes sequências de realização. Na primeira metade (20 crianças), foi aplicado primeiramente este teste da maneira convencional, em cabina acústica e com fones auriculares. Posteriormente, após 15 dias de intervalo (tempo sugerido por KeITH (12)), reaplicou-se então o teste, nas mesmas crianças, porém, em campo livre, sendo este denominado Grupo em estudo um (G1); e na outra metade ( 20 crianças) o processo foi o inverso, primeiramente aplicou-se o THAAS em campo livre, e após o intervalo de tempo mínimo de 15 dias, o teste foi aplicado novamente, porém, dessa segunda vez em cabina acústica, com fones auriculares, determinado como Grupo em estudo dois (G2) (Figura 1).

O protocolo de investigação foi composto do teste comportamental (Teste de Habilidade de Atenção Auditiva Sustentada - THAAS) que avalia a habilidade de atenção auditiva sustentada, apresentado em duas formas, com fones auriculares (modo convencional) e em campo livre.

O THAAS (6) consiste em um método de informação objetiva para descrever e avaliar o comportamento de

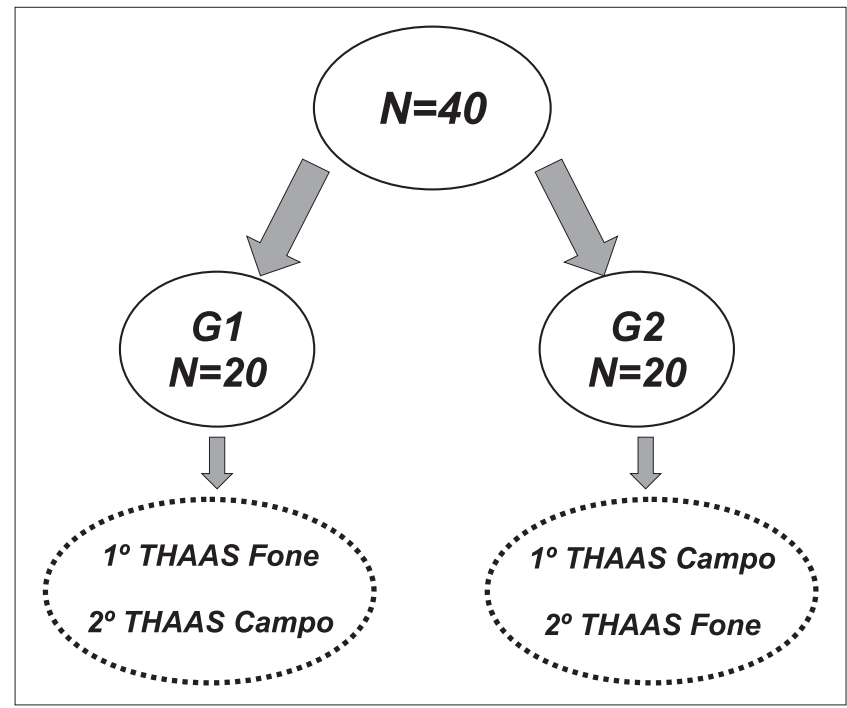

Figura 1. Esquema de distribuição de crianças na pesquisa e formação dos grupos.

atenção auditiva em crianças, por meio da avaliação da habilidade da criança escutar estímulos auditivos durante um período de tempo prolongado e de responder somente para um estímulo específico. É uma tarefa de vigilância auditiva, indicada pelas respostas corretas para as pistas linguísticas específicas, e serve para medir a atenção sustentada, indicada pela habilidade da criança em manter a atenção e concentração na tarefa por um período de tempo prolongado. Consistem na apresentação binaural e diótica de uma lista de 21 palavras monossilábicas (pé, sim, flor, gol, trem, mar, sol, quer, mal, lã, boi, meu, sal, pai, gás, vou, céu, não, já, pó e um), as quais são repetidas e rearranjadas aleatoriamente, formando uma lista de 100 palavras incluindo as 20 ocorrências da palavra alvo "não". Esta lista, gravada em CD, é apresentada seis vezes ininterruptamente, totalizando, dessa forma, 600 palavras monossilábicas, com uma duração de 10 minutos.

A apresentação do teste se deu a uma intensidade de 50 dBNS, considerando a média dos limiares aéreos auditivos $(500 \mathrm{~Hz}, 1 \mathrm{kHz}$ e $2 \mathrm{kHz})$ de cada orelha.

Cada criança foi instruída oralmente pela avaliadora de que ouviria uma lista de palavras e que deveria levantar a mão toda vez que ouvisse a palavra "não".

Anteriormente à primeira a presentação da lista de 600 palavras do teste THAAS, foi apresentada à criança, para prática, uma amostra gravada em CD, de 50 palavras monossilábicas e apresentadas, também, ininterruptamente, sendo 10 delas a palavra "não", disposta de maneira aleatória. Somente após a criança ter entendido a tarefa, o teste foi então aplicado. 
A aplicação do teste se deu por meio de um Disc Man 40+séc. virtual antishockda Toshiba, com saída line out, acoplado a um audiômetro, o Audiômetro SD 50 para aplicação com fones auriculares, e o audiômetro Clinical Audiometer AC40 para aplicação em campo livre.

Para aplicação do THAAS em campo livre, cuidado com relação ao ambiente tratado acusticamente foi preestabelecido e mantido os ângulos de incidência do sinal em todas as medidas, sendo que a criança foi posicionada a $60^{\circ}$ Azimute das duas caixas de som lateralmente, com distância de 2 metros, formando um triângulo equilátero, de maneira que a incidência do som foi a mesma para ambas as orelhas; além de o pesquisador ter-se mantido atento às condições físicas e psicológicas da criança durante o teste (Figura 2).

Os dados foram analisados tendo em vista as respostas obtidas no THAAS com fones auriculares em cabina acústica e em campo livre.

Para determinar o desempenho da criança neste teste, foram considerados os erros de desatenção, quando a criança não levantou a mão em resposta à palavra alvo ("não"); erros de impulsividade, quando a criança levantar a mão para outra palavra ao invés da palavra alvo ("não"); a contagem do número de erros de desatenção acrescida do número de erros de impulsividade permitiu obter a pontuação total de erros do THAAS. A vigilância foi obtida calculando-se o número de respostas corretas para a palavra "não" durante a sequência das seis a presentações. O cálculo dessa medida foi necessário, a fim de se verificar o decréscimo da vigilância, ou seja, o declínio na atenção que ocorreu com o tempo durante a tarefa de vigilância, que será obtida calculando-se o número de respostas corretas para a palavra "não" na 1a apresentação subtraindo-se o número de respostas corretas para a 6ª apresentação. A diferença encontrada entre esses dois números é o que se denomina decréscimo de vigilância.

Seguindo dados normativos (6) para a idade de sete anos o valor médio esperado para os erros de desatenção é 31, para a impulsividade é oito, para a pontuação total é 36 e para o decréscimo de vigilância um valor menor que oito.

Para a análise dos resultados, adotou-se o caráter duplo cego, não sendo possível ao pesquisador identificar a maneira de aplicação do teste (com fones ou em campo livre) a fim de se evitar que os resultados tenham sido viciados ou tendenciosos.

Seguindo o objetivo proposto, os resultados do THAAS foram analisados e computados de modo a se realizar posteriormente comparação dos dados obtidos intra-sujeitos (com as mesmas crianças) em dois momen-

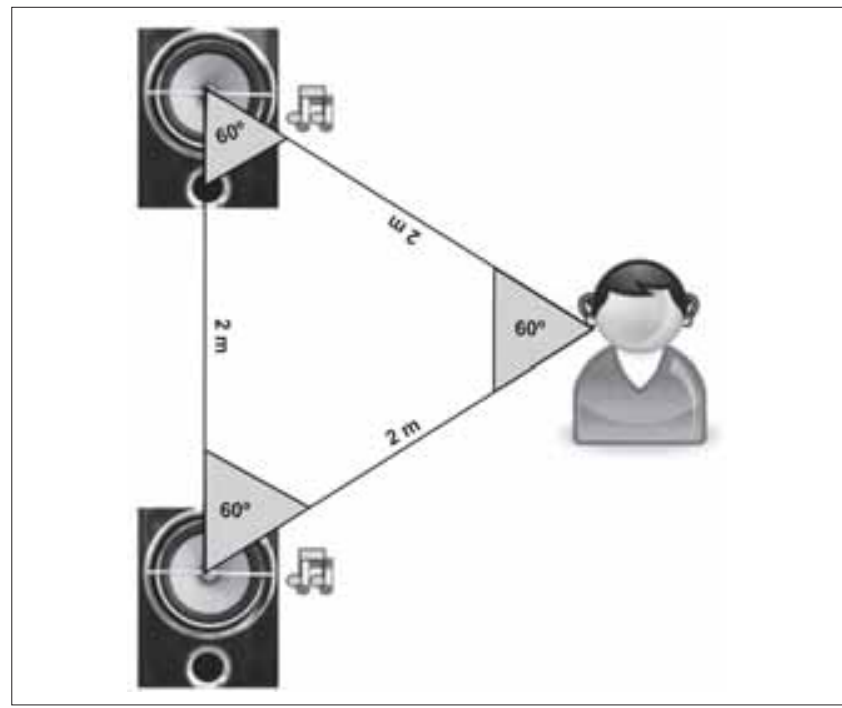

Figura 2. Esquema de avaliação em campo livre.

tos: $1^{\circ}$ na apresentação com fones auriculares em cabina acústica e $2^{\circ}$ em campo livre (não necessariamente nesta mesma ordem).

Para avaliar o desempenho entre os grupos e gênero, os testes estatísticos utilizados foram a Análise de Variância (ANOVA) e o teste Tukey. Para estas análises, utilizou-se o CD Pacotico, versão 4.6 (16).

As variáveis quantitativas foram representadas por média, mediana, desvio padrão e valores mínimo e máximo. Em todos os testes, o nível de rejeição da hipótese de nulidade adotado foi de $5 \%(\mathrm{p}<0,05)$.

\section{Resultados}

Seguindo os objetivos propostos, a Tabela 1 apresenta o desempenho (média e desvio padrão) das crianças dos dois grupos amostrados, na aplicação do THAAS na situação com fones auriculares e em campo livre.

A análise de variância a três critérios não apresentou diferença significativa entre os gêneros em nenhuma das condições ou grupos ( $p>0,05)$. Por isso passou-se a analisar os dados sem separação entre gêneros.

A Tabela 2 apresenta a comparação entre formas na mesma condição quando aplicados na primeira vez, e a Tabela 3, quando aplicados na segunda vez.

Assim, quando aplicados pela primeira vez só houve diferença entre fone e campo na desatenção $(p=0,049)$, não havendo diferença na segunda aplicação. 
Tabela I. Desempenho geral pelo THAAS por condição de aplicação (Fone ou Campo), gênero e grupo.

\begin{tabular}{|c|c|c|c|c|c|c|c|c|c|}
\hline \multirow[t]{3}{*}{ Forma } & \multirow[t]{3}{*}{ Comportamento } & \multicolumn{4}{|c|}{$\mathrm{Gl}$} & \multicolumn{4}{|c|}{ G2 } \\
\hline & & \multicolumn{2}{|c|}{ Feminino } & \multicolumn{2}{|c|}{ Masculino } & \multicolumn{2}{|c|}{ Feminino } & \multicolumn{2}{|c|}{ Masculino } \\
\hline & & média & $\mathrm{dp}$ & média & $\mathrm{dp}$ & média & $\mathrm{dp}$ & média & $d p$ \\
\hline Fone & $\begin{array}{l}\text { Desatenção } \\
\text { Impulsividade } \\
\text { Total de erros } \\
\text { Decréscimo de vigilância }\end{array}$ & $\begin{array}{c}17,2 \\
3,7 \\
20,9 \\
1,5\end{array}$ & $\begin{array}{c}10,7 \\
2,2 \\
10,9 \\
1,6\end{array}$ & $\begin{array}{c}15,4 \\
4,0 \\
19,4 \\
1,6\end{array}$ & $\begin{array}{l}6,4 \\
2,0 \\
7,4 \\
2,1\end{array}$ & $\begin{array}{c}11,9 \\
1,3 \\
13,2 \\
1,7\end{array}$ & $\begin{array}{l}8,9 \\
2,1 \\
9,7 \\
1,6\end{array}$ & $\begin{array}{c}7,0 \\
4,4 \\
11,4 \\
0,7\end{array}$ & $\begin{array}{l}9,1 \\
4,9 \\
9,6 \\
1,9\end{array}$ \\
\hline Campo & $\begin{array}{l}\text { Desatenção } \\
\text { Impulsividade } \\
\text { Total de erros } \\
\text { Decréscimo de vigilância }\end{array}$ & $\begin{array}{c}9,9 \\
3,7 \\
13,6 \\
0,7\end{array}$ & $\begin{array}{c}10,6 \\
2,2 \\
10,8 \\
1,2\end{array}$ & $\begin{array}{l}4,6 \\
4,2 \\
8,8 \\
0,0\end{array}$ & $\begin{array}{l}2,9 \\
4,2 \\
6,1 \\
0,5\end{array}$ & $\begin{array}{c}12,3 \\
4,0 \\
16,3 \\
2,3\end{array}$ & $\begin{array}{l}8,7 \\
2,6 \\
8,7 \\
2,8\end{array}$ & $\begin{array}{c}9,8 \\
6,0 \\
15,8 \\
2,7\end{array}$ & $\begin{array}{l}6,0 \\
6,2 \\
10,3 \\
2,1\end{array}$ \\
\hline
\end{tabular}

Tabela 2. Comparação entre Fone e Campo quando os mesmos são aplicados na primeiravez.

\begin{tabular}{lcccccc}
\hline Medida & \multicolumn{2}{c}{ GI Fone } & \multicolumn{2}{c}{ G2 Campo } & dif. & $P$ \\
& média & dp & média & dp & & \\
\hline Desatenção & 16,4 & 8,8 & 11,2 & 7,5 & $-5,3$ & $0,049 *$ \\
Impulsividade & 3,9 & 2,1 & 4,9 & 4,6 & 1,1 & $0,356 \mathrm{~ns}$ \\
Total de erros & 20,3 & 9,3 & 16,1 & 9,2 & $-4,2$ & $0,159 \mathrm{~ns}$ \\
Decréscimo de vigilância & 1,5 & 1,8 & 2,5 & 2,5 & 1,0 & $0,175 \mathrm{~ns}$ \\
\hline
\end{tabular}

*-diferença estatisticamente significativa $(p<0,05)$

ns - diferença estatisticamente não significativa

Tabela 3. Comparação entre Fone e Campo quando os mesmos são aplicados na segundavez.

\begin{tabular}{lcccccc}
\hline Medida & \multicolumn{2}{c}{ GI Fone } & \multicolumn{2}{c}{ G2 Campo } & dif. & $P$ \\
& média & dp & \multicolumn{1}{c}{ média } & dp & & \\
\hline Desatenção & 7,5 & 8,4 & 9,7 & 9,1 & 2,2 & $0,433 \mathrm{~ns}$ \\
Impulsividade & 4,0 & 3,2 & 2,7 & 3,9 & $-1,3$ & $0,271 \mathrm{~ns}$ \\
Total de erros & 11,5 & 9,1 & 12,4 & 9,4 & 1,0 & $0,747 \mathrm{~ns}$ \\
Decréscimo de vigilância & 0,4 & 1,0 & 1,3 & 1,7 & 0,9 & $0,066 \mathrm{~ns}$ \\
\hline
\end{tabular}

*-diferença estatisticamente significativa $(p<0,05)$

ns - diferença estatisticamente não significativa

Tabela 4. Comparação entre Campo quando aplicado primeiro (G2)com Campo quando aplicado em segundo lugar (GI).

\begin{tabular}{|c|c|c|c|c|c|c|}
\hline \multirow[t]{2}{*}{ Medida } & \multicolumn{2}{|c|}{ G2 Campo } & \multicolumn{2}{|c|}{ Gl Campo } & \multirow[t]{2}{*}{ dif. } & \multirow[t]{2}{*}{$P$} \\
\hline & média & $\mathrm{dp}$ & média & $d p$ & & \\
\hline Desatenção & 11,2 & 7,5 & 7,5 & 8,4 & $-3,7$ & $0,156 \mathrm{~ns}$ \\
\hline Impulsividade & 4,9 & 4,6 & 4,0 & 3,2 & $-1,0$ & $0,450 \mathrm{~ns}$ \\
\hline Total de erros & $\mid 6,1$ & 9,2 & 11,5 & 9,1 & $-4,6$ & $0,120 \mathrm{~ns}$ \\
\hline Decréscimo de vigilância & 2,5 & 2,5 & 0,4 & 1,0 & $-2,1$ & $0,00 \mid *$ \\
\hline
\end{tabular}

*-diferença estatisticamente significativa $(p<0,05)$

ns - diferença estatisticamente não significativa

Comparação também foi realizada entre primeira e segunda vez, na mesma forma de aplicação (Tabelas 4 e 5).

Para a condição em campo livre houve uma tendência geral de diminuição dos valores na segunda vez, porém com significância estatística somente para o decréscimo de vigilância $(\mathrm{p}=0,001)$.
Para na condição com Fone também houve uma tendência geral de diminuição dos valores na segunda vez, porém com significância estatística somente para a desatenção $(p=0,024)$ e para Total de erros $(p=0,011)$.

No que se refere ao intervalo de tempo percorrido entre a aplicação do THAAS em fone e em campo no G1 
Tabela 5. Comparação entre Fone quando aplicado primeiro (GI) com Fone quando aplicado em segundo lugar (G2).

\begin{tabular}{lcccccc}
\hline Medida & \multicolumn{2}{c}{ GI Fone } & \multicolumn{2}{c}{ G2 Fone } & dif. & P \\
& média & $d p$ & média & $d p$ & & \\
\hline Desatenção & 16,4 & 8,8 & 9,7 & 9,1 & $-6,7$ & 0,024 * \\
Impulsividade & 3,9 & 2,1 & 2,7 & 3,9 & $-1,2$ & 0,249 ns \\
Total de erros & 20,3 & 9,3 & 12,4 & 9,4 & $-7,9$ & $0,01 \mid$ * \\
Decréscimo de vigilância & 1,5 & 1,8 & 1,3 & 1,7 & $-0,3$ & 0,660 ns \\
*-diferença estatisticamente significativa(p<0,05) & & & \\
ns-diferença estatisticamente nãosignificativa &
\end{tabular}

e no G2, um máximo de 360 e um mínimo de 25 dias para o G1 e 75 e 15 dias para o G2 foi verificado, mostrandose mais heterogênea para o G1 e mais homogênea para o G2.

\section{DISCUSSÃO}

A ausência de diferença significante encontrada para os gêneros está concordante com trabalhos encontrados na literatura específica $(17,18,6,8,19)$. Por outro lado, pesquisas demonstram melhor desempenho do gênero feminino quando comparado ao masculino (20-24), fato esse explicado devido à maior dificuldade de diagnosticar crianças do gênero feminino para o déficit atencional, por apresentarem comportamentos menos exacerbados do que o gênero masculino (25). Pesquisas referem (26) que a habilidade de atenção auditiva de mulheres é melhor porque elas conseguem mantê-la, mesmo para um estímulo sem significado como o ruído. Outros estudos $(27,28)$ encontraram pior desempenho para o gênero feminino. Autores (20) descreveram, ainda, que a diferença com relação à atenção entre os gêneros diminui com o avanço da idade.

O THAAS é um teste nacional baseado em um teste americano, o Auditory Continuous Performance Test (ACPT), utilizado em uma série de estudo clínicos (29-31,13, 32-34). KeIth (12) verificou por meio deste teste ( $A C P T)$ que os erros de desatenção foram três vezes mais frequentes que os erros de impulsividade. Feniman (6) encontrou proporção média de 3,5. No presente estudo no G1 a proporção encontrada para os erros de desatenção comparados aos de impulsividade foi similar, exceto para a situação de aplicação em campo livre no gênero masculino, no qual foi verificada média de erros de desatenção igual a 4,6 e de erros de impulsividade igual a 4,2 (Tabela 1$)$.

A diferença encontrada entre o G1 e o G2 (com relação aos valores obtidos somando-se as médias do número de erros para fone e campo) possivelmente deve se à diferença do intervalo de tempo percorrido entre as duas formas de aplicação.

Embora em ambos os grupos o desempenho das crianças tenha sido melhor na segunda apresentação do teste, comparando o desempenho do G1 ao G2 com fone e em campo livre (Tabela 1), considerando as duas apresentações, os escores do G2 tiveram valores mais próximos que no G1, o que poderia ser explicado baseando-se na hipótese da diferença de intervalo de tempo nos dois grupos, ou, também, pode ser que tenha sido ao acaso.

Baseando-se nos resultados obtidos, na comparação entre os grupos, observou-se que o G1 teve maior número de erros que o G2 na aplicação com fone para todos os itens do THAAS, sendo estatisticamente significativo para desatenção e total de erros.

Com base nos achados do G1, poder-se-ia pensar que a situação de aplicação do teste em campo livre atuou como facilitador do teste, por ter sido verificado melhor desempenho das crianças. Assim sendo, a cabina acústica utilizada para aplicação do teste com fones foi motivo de distração nas crianças, pois havia menos contato visual com o avaliador nessa situação, diferentemente na situação em campo livre em que há maior proximidade na situação, uma vez que o ambiente acusticamente tratado era o mesmo para o avaliador e para a criança. Na aplicação com fones, a criança foi avaliada em uma cabina acústica, na qual o avaliador encontrava-se do lado de fora, observando a criança apenas por um pequeno visor de vidro, o que poderia diminuir a motivação da criança durante a situação de avaliação.

No entanto, como no G2, no qual a aplicação do THAAS ocorreu primeiramente em campo, e no segundo momento com fone e as crianças apresentaram melhor desempenho com fone do que em campo, observou-se o mesmo padrão de desempenho (melhor na segunda aplicação) - Tabelas 2 e 3 . 
O mesmo pode ser pensado para a comparação entre os grupos para aplicação do THAAS em campo livre, uma vez que o G2 apresentou mais erros que o G1 para todos os itens do teste (sendo essa diferença estatisticamente significativa para o decréscimo de vigilância). Valese ressaltar que no $\mathrm{G} 2$ a primeira aplicação foi em campo livre, e no G1 foi a segunda aplicação que ocorreu em campo livre, em que as crianças poderiam já estar familiarizadas com o teste.

Na realização do teste - reteste do $A C P T$ obtida por KeITH (12), este não verificou significante diferença estatística entre a primeira e a segunda vez em que seu teste foi aplicado (intra-sujeitos). Esta proximidade entre os escores encontrados no reteste dão maior confiabilidade ao teste. Ressalta-se, ainda, que as duas aplicações do teste no trabalho de Kегтн (12) foram obtidas com a mesma forma de apresentação (apenas com fones), diferentemente da presente pesquisa.

Esses dados estão em concordância com os achados de pesquisadores (18) que verificaram um desempenho médio melhor na segunda apresentação de uma lista que avaliava atenção seletiva, acreditando que esse fato devase ao efeito de aprendizagem.

Em termos neurais, o desempenho de certas atividades treinadas previamente (ou talvez de forma mais ampla, o contexto) deve pré-ativar redes as neurais, de modo que o fruto de seu processamento passe a ter prioridade para os sistemas atencionais (35).

A utilização de um processo randômico ou aleatório a fim de conceder aos participantes de um determinado estuda a mesma probabilidade de receber a intervenção a ser testada ou o seu controle, revolucionou a prática clínica a o ter sido utilizado pela primeira vez no final da década de 40. Mais recentemente, os ensaios clínicos randomizados vêm sendo descritos como o "padrão-ouro" na avaliação de questões terapêuticas em saúde. Por meio desse tipo de estudo reduz-se a probabilidade de obter dados tendenciosos na pesquisa.

O princípio de randomização é simples, e se baseia no fato de que os participantes de um determinado estudo tenham a mesma probabilidade de receber tanto a intervenção a ser testada quanto o seu controle (36). Se esse princípio é respeitado e realizado de maneira adequada, a randomização reduz o risco de erros sistemáticos (ou viés), produzindo um equilíbrio entre os diversos fatores de risco que podem influenciar no desfecho clínico a ser medido (37).

Acredita-se que a randomização desse estudo foi o processo que consentiu esses achados, permitindo-se con- cluir sobre o efeito de aprendizagem, como demonstraram os resultados.

No entanto, autores (38), estudando a atenção em crianças, verificaram que com a repetição do teste as crianças que foram melhores num primeiro momento, tornaram-se mais impulsivas e menos atentas na segunda apresentação, sendo discordante com os resultados verificados tanto para o G1 como para o G2, visto um melhor desempenho na segunda aplicação do teste, justificandose pelo efeito da aprendizagem.

Concordando com os resultados deste trabalho, a aplicação do P300 mostrou-se viável em campo livre, não apresentando diferenças estatisticamente significantes para o gênero e modo de apresentação do teste (39).

\section{ConClusão}

O desempenho de crianças no THAAS mostrou-se similar nas diferentes formas de apresentação aplicadas, no entanto, escores melhores foram observados na segunda apresentação.

As crianças do gênero feminino e do masculino não diferiram nos escores do THAAS para as duas formas de apresentação.

O teste demonstrou aplicabilidade em ambas as formas.

\section{REFERÊNCIAS BIBLIOGRÁFICAS}

1. US Preventive Services Task Force. Universal screening for hearing loss in newborns: US Preventive Recommendation Statement. Pediatrics. 2008, 122(1):1438.

2. Manrique MJ, Huarte A. Hipoacusias. Surdez. In: ChevrieMuller C, Narbona J. A linguagem da criança: aspectos normais e patológicos. 2 $2^{\mathrm{a}}$ ed. Porto Alegre: Artmed; 2005 , p:264-69.

3. Raz A, Buhle J. Typologies of attentional networks. Nature Reviews. 2006, 7:367-79.

4. Lima RF. Compreendendo os mecanismos atencionais. Ciências e Cognição. 2005, 6:113-22.

5. Lima RF, Tabaquim MLM, Ciasca SM. Sistema atencional e funções executivas na infância. In: Ciasca SM, Rodrigues SD, Salgado CA. TDHA: transtorno de déficit de atenção e hiperatividade. Rio de Janeiro: Revinter; 2010:1-20. 
6. Feniman MR. Aplicação do teste de atenção auditiva FC2 em crianças ouvintes normais [tese]. Bauru: Universidade de São Paulo - Faculdade de Odontologia de Bauru; 2004.

7. Feniman MR, Ortelan RR, Campos CF, Cruz MS, Lauris JRP. A Habilidade de Atenção Auditiva Sustentada em Crianças. ACTA ORL/Técnicas em Otorrinol. 2007, 25(4): 280-284.

8. Mondelli MFCG, Carvalho FRP, Feniman MR, Lauris JRP. Perda auditiva leve: desempenho no Teste da Habilidade de Atenção Auditiva Sustentada. Pró-Fono Rev de Atual Cient. 2010, 22(3):245-50.

9. Lemos ICC, Feniman MR. Teste de Habilidade de Atenção Auditiva Sustentada (THAAS) em crianças de sete anos com fissura labiopalatina. Braz. J. Otorhinolaryngol. 2010, 76(2):199-205.

10. Souza SRB. Avaliação as atenção e memória operacional fonológica em indivíduos com síndrome velocardiofacial. [dissertação]. Bauru: Universidade de São Paulo - Faculdade de Odontologia de Bauru; 2009.

11. Duarte TF, Maximino LP, Feniman MR (in press 2011). A Habilidade de atenção auditiva sustentada em crianças com fissura- labiopalatina e transtorno fonológico. Revista Sociedade Brasileira de Fonoaudiologia.

12. Keith RW. ACPT: Auditory continuous performance test. San Antonio, TX, Psychological Corporation, 1994.

13. Tillery KL, KatzJ, Keller WD. Effects of Methylphenidate (Ritalin) on auditory performance in children with attention and auditory processing disorders. J Speech, Lang Hear Res. 2000, 43(4):893-901.

14. Jerger J. Clinical experience with impedance audiometry. Arch Otolaryngol. 1970, 92:3111-24.

15. Northern JL, Downs MP. Audição em crianças. São Paulo: Manole; 1989.

16. Lopes ES. Pacotico, versão 4.6: pacotinho estatístico [CDROM]. Bauru: Faculdade de Odontologia de Bauru, Universidade de São Paulo; 2007.

17. Seidel WT, Joschko M. Evidence of difficulties in sustained attention in children with ADDH. J Abnorm Child Psychol. 1990, 18(2):217-29.

18. Chermak GD, Montgomery MJ. Form equivalent of selective auditory attention test administered to 6-year-old children. ASHA. 1992, (35):661-5.
19. Picolini MM, Stivanin D, Sampaio AR, Salvador KK, Lauris JRP, Feniman MR. Atenção Auditiva: Período do Dia e Tipo de Escola. Arq. Int. Otorrinolaringol. / Intl. Arch. Otorhinolaryngol. 2010, 14(2):174-79.

20. Gale A, Lynn R. A developmental study of attention. Br J Educ Psychol. 1972, 42(3):260-6.

21. Brito GNO, Pinto RCA, Lins MFC. A behavioral assessment scale for attention deficit disorder in brazilian children based on DSM-IIIR criteria. J Abnorm Child Psychol. 1995, 23(4):509-20.

22. Pineda D, Ardila A, Rosselli M, Arias BE, Henao GC, Gomes LF, et al. Prevalence of. Attention-deficit/hyperactivity disorders symptoms in 4- to 17-year-old children in the general population. J Abnorm Child Psychol. 1999, 27(6):455-62.

23. Hartung CM, Willcutt EG, Lahey BB, Pelham WE, Loney J, Stein MA, Keenan K. Sex differences in young children who meet criteria for attention deficit hyperactivity disorder. J Clin Child Adolesc Psychol. 2002, 31(4):45364.

24. Groot AS, Sonneville LMJ, Stins JF, Boomsma DI. Familial influences on sustained attention and inhibition in preschoolers. J Chil Psychol Psychiat. 2004, 45(2):306-14.

25. Rhee SH, Waldman ID. Etiology of sex differences in the prevalence of ADHD: an examination of inattention and hyperactivity-impulsivity. Am J Med Genet. 2004, 127B(1):60-4.

26. QueirozDS, Momensohn-Santos M. Diferenças funcionais entre o córtex auditivo primário de homens e mulheres. Rev Distúrb Comun. 2009, 21(1): 107-9.

27. Lin CCH, Hsiao CK, Chen WJ. Development of sustained attention assessed using the continuous performance test among children 6-15 years of age. J Abnorm Child Psychol. 1999, 27(5):403-12.

28. Biederman J, Mick E, Faraone SV, Braaten E, Doyle A, Spencer T, Wilens TE, Frazier E, Johnson MA. Influence of gender on attention deficit hyperactivity disorder in children referred to a psychiatric clinic. Am J Psychiatry. 2002, 159(1):36-42.

29. McGee R, Williams S, Feehan M. Attention deficit disorder and age of onset of problems behaviors. J Abnorm Child Psychol. 1992, 20(5):487-502.

30. Riccio CA, Cohen MJ, Hynd GW, Keith RW. Validity of the Auditory Continuous Test in differentiating central 
processing auditory disorders with and withot ADHD. J Learn Disabil. 1996, 29:561-6.

31. Feniman MR. Central auditory processing disorder in attention deficit hyperactivity disorder, and language-based learning impairment: a normative study. Cincinnati University of. Cincinnati, 1997. (Relatório final CNPq período Dezembro/96 à Novembro/97 - processo no 201175/96-7 (NV).

32. Medwetsky L. Central auditory processing testing: a battery approach. In: Katz J, Burkard RF, Medwetsky L. eds. Handbook of Clinical Audiology. Baltimore, Williams \& Wilkins, 2002. p.510-24.

33. Hagelthorn KM, Hiemenz JR, Pillion JP, Mahone EM. Age and task parameters in continuous performance tests for preschoolers. Percept Mot Skills. 2003, 96 (3 Pt 1):97589 .

34. Mahone ME. Measurement of attention and related functions in the preschool child. Ment Retard Dev Disabil Res Rev. 2005, 11(3):216-25.
35. Helene AF, Xavier FG. A construção da atenção a partir da memória. Rev Bras Psiquiatr. 2003, 25(Supl II):12-20.

36. Yusuf S, Collins R, Peto R. Why do we need some large, simple randomized trials? Statistics in Medicine. 1984, 3:40920.

37. Collins R, Peto R, Gray R, Parish S. Large-scale randomized evidence: trials and overviews. In: Weatherall DJ, Ledingham JGG, Warrell DA. editors. Oxford Textbook of Medicine. 3rd ed. Oxford: Oxford University Press. 1996, 21-32.

38. Weissberg R, Ruff HA, Lawson KR. The usefulness of reaction time tasks in studying attention and organization of behavior in young children. J Dev Behav Pediatr. 1990, 11(2):59-64.

39. Duarte J L, Alvarenga KF, Costa OA. Potencial cognitivo p300 realizado em campo livre: aplicabilidade do teste. Rev Bras Otorrinolaringol. 2004, 70(6):780-5. 